\title{
Flow-Induced Crystallization of Collagen: A Potentially Critical Mechanism in Early Tissue Formation
}

\author{
Jeffrey A Paten ${ }^{\dagger}$, Seyed Mohammad Siadat ${ }^{\dagger}$, Monica E. Susilo ${ }^{\dagger}$, Ebraheim N. Ismail ${ }^{\dagger}$, \\ Jayson L. Stoner ${ }^{\dagger}$, Jonathan P. Rothstein ${ }^{\ddagger}$, and Jeffrey W. Ruberti ${ }^{\dagger}$ \\ tDepartment of Bioengineering, Northeastern University, 360 Huntington Avenue, Boston, \\ Massachusetts 02115, United States \\ FDepartment of Mechanical and Industrial Engineering, University of Massachusetts Amherst, \\ 160 Governors Drive, Amherst, Massachusetts 01003, United States
}

\section{Abstract}

The type I collagen monomer is one of nature's most exquisite and prevalent structural tools. Its $300 \mathrm{~nm}$ triple-helical motifs assemble into tough extracellular fibers that transition seamlessly across tissue boundaries and exceed cell dimensions by up to 4 orders of magnitude. In spite of extensive investigation, no existing model satisfactorily explains how such continuous structures are generated and grown precisely where they are needed (aligned in the path of force) by discrete, microscale cells using materials with nanoscale dimensions. We present a simple fiber drawing experiment, which demonstrates that slightly concentrated type I collagen monomers can be "flow-crystallized" to form highly oriented, continuous, hierarchical fibers at cell-achievable strain rates $\left(<1 \mathrm{~s}^{-1}\right)$ and physiologically relevant concentrations $(\sim 50 \mu \mathrm{M})$. We also show that application of tension following the drawing process maintains the structural integrity of the fibers. While mechanical tension has been shown to be a critical factor driving collagen fibril formation during tissue morphogenesis in developing animals, the precise role of force in the process of building tissue is not well understood. Our data directly couple mechanical tension, specifically the extensional strain rate, to collagen fibril assembly. We further derive a "growth equation" which predicts that application of extensional strains, either globally by developing muscles or locally by fibroblasts, can rapidly drive the fusion of already formed short fibrils to produce longrange, continuous fibers. The results provide a pathway to scalable connective tissue manufacturing and support a mechano-biological model of collagen fibril deposition and growth in vivo.

Correspondence to: Jeffrey W. Ruberti.

Associated content: Supporting Information: The Supporting Information is available free of charge on the ACS Publications website at DOI: $10.1021 / a c s n a n o .5 b 07756$.

Author Contributions: J.A.P. performed the fiber drawing experiments, analyzed the data, and prepared the manuscript. S.M.S. performed the viscosity/concentration experiments, the extensional strain rate/fluorescence experiments, the negative control experiments and analyzed the data. M.E.S. analyzed the fibril banding periodicity and contributed to the experimental design. E.N.I. performed the QFDE experiments used to observe the droplet surface with TEM. J.L.S. assisted with the fiber drawing experiments. J.P.R. analyzed the fiber drawing videos to determine the extensional strain and contributed to the FIC section of the manuscript. J.W.R. contributed to the experimental design and prepared the manuscript.

Notes: The authors declare no competing financial interest. 


\section{Graphical abstract}

Collagen Flow Induced Crystallization Occurs Under Physiologically Relevant Conditions

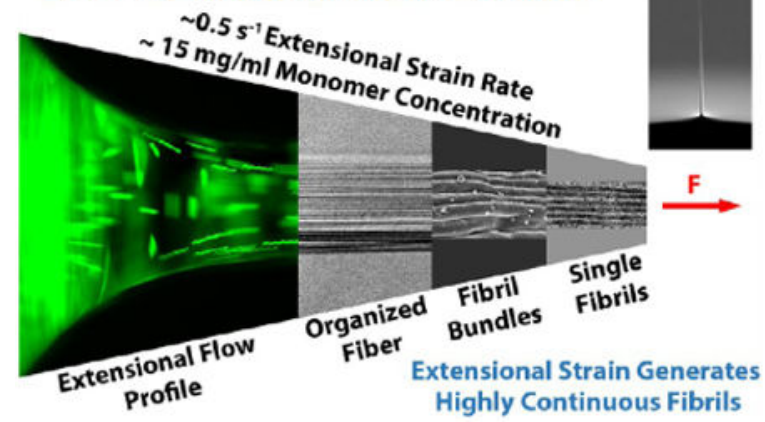

\section{Keywords}

collagen; self-assembly; development; extensional strain; flow-induced crystallization

Fibril-forming collagens are the principal structural building blocks of the extracellular matrix (ECM), as demonstrated by their broad prevalence across animal phyla. ${ }^{1}$ The collagen motif arose in a premetazoan class of protists (Choanoflagellata), ${ }^{2}$ known for their extracellular structures. ${ }^{3}$ Thus, collagen has served as a structural molecule for approximately one billion years. Its amino acid composition has been tuned by retention of numerous, beneficial mutations, duplications, and substitutions ${ }^{4}$ to permit entropy-driven fibril assembly. ${ }^{5}$ While many collagen types have evolved, ${ }^{4}$ the fibril-forming collagens in vertebrates (type I, II, III, V, XI) are the primary proteins responsible for bearing loads in blood vessel, bone, cartilage, cornea, intervertebral disk, ligament, sclera, skin, and tendon. Degeneration of or damage to collagenous load-bearing structures such as intervertebral disks, ${ }^{6}$ anterior cruciate ligaments, ${ }^{7,8}$ and cartilage ${ }^{9}$ continues to significantly diminish the quality of life for millions of individuals. Tendon ruptures, aortic aneurysms, and keratoconus in the cornea are ultimately due to collagen network failures. For all of these conditions, there are few satisfactory clinical responses. In the last few decades, tissue engineers have been attempting to recapitulate collagenous structures to affect in situ repair of degenerated tissue.

In spite of the fact that collagen solutions have been induced to self-assemble into nativelike, banded fibrils in vitro since the $1950 \mathrm{~s},{ }^{10}$ experimentally advancing to the generation of highly aligned fibrils within a larger fiber or lamella has been slow. Attempts have been made to control collagen structure using contact guidance, ${ }^{11}$ electrochemically induced alignment, ${ }^{12}$ electrospinning, ${ }^{13}$ extrusion, ${ }^{14}$ flow, ${ }^{15}$ liquid-crystalline-induced alignment, ${ }^{16}$ and magnetism, ${ }^{17}$ but the final products still suffer from numerous shortcomings. None of the techniques produce structures that satisfy all of the following conditions: appropriate dimensions, reasonable manufacturing speeds, native tissue organization, and precise alignment control. Further, little effort has been made to tie most of these approaches to the processes which are responsible for the in vivo generation of collagenous load-bearing structures. This is likely due to our poor understanding of how mesenchymal cells actually 
deposit and organize collagen over long distances. While there has been speculation about the role of liquid-crystal collagen phasing as a mechanism of collagen fibril organizational control during morphogenesis, ${ }^{18}$ the most widely accepted idea is that proposed by Birk and Trelstadt ${ }^{19}$ and carried forward by Kadler and coworkers, ${ }^{20}$ which suggests that cells directly deposit assembled collagen via structures referred to as "fibripositors". Recently, however, it has been demonstrated that mechanical tension, as opposed to fibril extrusion, is critical to the process of collagen deposition and organizational control. ${ }^{21-23}$

The accumulation of collagen, precisely in the path of tensile force, led us to consider the idea that tensile mechanical signals, which are robust and operate across all length scales, are "direct" drivers of collagen assembly and retention. ${ }^{24}$ Specifically, we hypothesized that mechanically sensitive mechanisms exist in the relationship between the collagen molecules themselves that promote the formation of structure in the path of force. These mechanisms most likely operate in the form of strain-driven molecular allostery, ${ }^{25,26}$ which alters fibril nucleation, association rate constants, and dissociation rate constants. ${ }^{27}$ In the mammalian ECM, it is already known that application of tensile mechanical strain accelerates the kinetics of fibronectin assembly, ${ }^{28}$ accelerates fibrin and elastin enzymatic degradation, ${ }^{29,30}$ slows collagen thermal denaturation ${ }^{31}$, and reduces collagen enzymatic proteolysis. ${ }^{32}$ In spiders and silkworms, the extensional strain rate achieved while flowing the silk dope to the spinneret appears to be critical for initiating fiber formation. ${ }^{33}$ From these observations, we hypothesized that physiologically relevant strains or strain rates, applied to collagen molecular aggregates, might initiate and/or enhance the assembly of highly aligned fibrils. Such a mechanism has the potential to conjure load-bearing connective tissues precisely where they are needed: in the path of force.

\section{Results}

We here show that organized collagen fibers can be assembled in the direction of low-level extensional strain rates. Fibers were formed by slowly drawing a microneedle from the enriched surface of a droplet comprising collagen monomers in solution (Figure 1A-D, Figure S1, and Movie S1). To isolate the location of the fiber initiation, the microneedle was maneuvered around the droplet surface after drawing a fiber. The attachment point of the fiber to the droplet exhibited a high degree of mobility (Movie S2), suggesting that assembly of the fiber was localized to the small "necking" region where the fiber attaches to the droplet. Figure 1E confirms that the initiation of the assembly of fibrils within the fiber was indeed confined to the necking region, where newly formed fibrils propagated back into the droplet only a short distance, revealing the precise location of fibril initiation. A thorough microscopic examination of the remaining droplet by DIC microscopy revealed no observable fibrillar structures.

The point of fibril nucleation in the drawn fiber was limited to an area where the geometry and fluid mechanics suggested the presence of an extensional strain field. Extensional strains are well-known to promote the crystallization of polymers via flow-induced crystallization (FIC) ${ }^{34}$ FIC promotes crystallization by two mechanisms: (1) increasing the number of nucleation sites and (2) accelerating crystallization kinetics. ${ }^{35}$ FIC is a direct produce FIC in aresult of the extensional-flow-induced stretching and aligning of polymer chains, which is 
known to lower the entropy of the assembling system and, as a consequence, reduces the change in free energy required for polymer crystallization to occur. ${ }^{36}$ Thus, collagen fibril assembly appears to proceed in direct analogy to FIC of polymers.

The extensional strain rate, $\dot{\varepsilon}$, was measured by tracking fluorescent beads during fiber drawing (Movie S3). The average $\dot{\varepsilon}$ was $0.39 \pm 0.18 \mathrm{~s}^{-1}$. The maximum observed $\dot{\varepsilon}$ was 0.7 $\mathrm{s}^{-1}$, well below the critical extensional strain rate, $\dot{\varepsilon}_{\mathrm{c}}=1 / \tau_{\mathrm{r}} \sim 7000 \mathrm{~s}^{-1}$ (where $\tau_{\mathrm{r}}$ is the relaxation time), required to align a single collagen monomer in an infinitely dilute solution.

37 The Weissenberg number, $W_{\mathrm{e}}$, balances the extensional strain rate with the relaxation time of a polymer: $W_{\mathrm{e}}=\dot{\varepsilon}_{\mathrm{c}} \tau_{\mathrm{r}}$. To produce FIC in a polymer solution, $W_{\mathrm{e}}$ should be in the range of 1.0 or greater. As can be seen, our extensional strain rate is far too low to produce a $W_{\mathrm{e}}$ near 1.0 in dilute solution. However, the droplet surface, from where the fibers were drawn, was far from infinitely dilute. A scaling analysis (detailed in the Supporting Information) that balanced the surface evaporation speed against collagen diffusion speed suggested that, within $45.8 \mu \mathrm{m}$ of the droplet surface, the average concentration should be $11.85 \mathrm{mg} / \mathrm{mL}$ just prior to drawing a fiber. To experimentally determine the concentration closer to the surface and the concentration gradient, we performed optical magnetic micro-viscometry on the droplet during evaporation in dry nitrogen (see Supporting Information). The data confirmed the existence of a steep concentration gradient from the droplet surface $(13.98 \pm 3.47$ $\mathrm{mg} / \mathrm{mL}$ ) to a point $200 \mu \mathrm{m}$ below the surface where the concentration was $4.6 \mathrm{mg} / \mathrm{mL}$ (the bulk droplet concentration). It should be noted that the fiber was drawn over a $\sim 2$ min time period. Thus, the exact surface concentration and viscosity were dynamically changing while the fiber was being created. The enriched surface was being depleted as monomers left the droplet and entered the fiber, yet further crowded as evaporation proceeded to occur.

Extrapolating from the concentration and viscosity relationship (presented in the Supporting Information Figure S5), we determined that the viscosity at the surface of the droplet was approximately $1660 \mathrm{cP}$ when fiber-pulling began. Under the assumption that the collagen monomer is a rigid rod, then in the dilute regime, the rotational relaxation time is known to scale linearly $(144 \mu \mathrm{s} / \mathrm{cP})$ with the viscosity of the fluid media. ${ }^{38}$ Thus, at the surface of the droplet, the rotational relaxation time of a collagen monomer was estimated to be $\tau_{\mathrm{r}}=0.24 \mathrm{~s}$ by scaling from its value in dilute aqueous solution. ${ }^{37}$ This suggests that a critical extensional strain rate of approximately $\dot{\varepsilon}_{\text {crit }}=1 / \tau_{\mathrm{r}}=4.2 \mathrm{~s}^{-1}$ is necessary to orient the collagen and induce crystallization. However, this estimate of relaxation time is too conservative because the droplet surface concentration was well above the overlap concentration of $\sim 2.5 \mathrm{mg} / \mathrm{mL},{ }^{39}$ making the solution semidilute. As a result, we expect that the high level of intermolecular associations in collagen increased the relaxation time and lowered the extensional strain rate necessary to cause molecular alignment. ${ }^{40}$ This expectation is supported strongly by rheological data obtained by Gobeaux et al. ${ }^{39}$ in their examination of acidic type I collagen in the semidilute regime $(\sim 16 \mathrm{mg} / \mathrm{mL})$. From their work, the relaxation time can be estimated either from the frequency of the crossover of the storage and loss moduli in the terminal regime or from the shear rate at which the shear viscosity begins to thin. Both of these approaches give an estimate of approximately $\tau_{\mathrm{r}}=5 \mathrm{~s}$ with an $\dot{\varepsilon}_{\text {crit }}=1 / \tau_{\mathrm{r}}=0.2 \mathrm{~s}^{-1}$, which is within the range of extensional strain rates measured during our fiber-pulling experiment. These independent measurements support our assertion 
that small extensional strain rates found in our experiment can result in collagen FIC at relatively low collagen concentrations $(\sim 15 \mathrm{mg} / \mathrm{mL})$.

Repair of fibers was readily demonstrated, as well. If a fiber was drawn from the droplet at too high a rate, it fractured at the necking region, and a short fiber segment remained attached to the glass needle (Figure 1F). The severed fiber could be "repaired" by merely reintroducing it into the droplet and redrawing at a lower rate (Figure 1G-I and Movie S4). Fiber assembly was reinitiated as the segment end was pulled through the solution, and a visible repair site was formed. Once the junction was created, the fiber continued to be generated without further defect. The data immediately suggest a potential route to repair collagen fibers in vivo via exogenous supply of monomer, in conjunction with the controlled application of tensile force.

Structural investigation of the drawn fibers with differential interference contrast (DIC) microscopy (Figure 2A,B) showed the high degree of fibrillar alignment and continuity within fibers drawn from either pepsin-extracted collagen monomers that lack telopeptides (atelo-) or acetic-acid-extracted collagen monomers with intact telopeptides (telo-). The slower rate of assembly for atelo-collagen ${ }^{41}$ may have permitted the finer organizational control observed in the atelo-fibers. This is possibly due to the increased time for molecular mobility within the growing collagen aggregates to more fully align fibrillar structures with the strain field. Collagen fibers generated from telo-collagen monomers typically possessed fibrils with a slight angular offset from the long axis of the fiber, which could be due to their relatively rapid assembly kinetics "setting" the structure early in the drawing process.

Regardless of the collagen source, fibers with remarkable internal fibrillar anisotropy were generated by drawing from a droplet of monomers. As a positive control for preassembly of the collagen prior to drawing, fibers were pulled from droplets containing observable, preassembled collagen networks (Figure 2C). The drawing of preassembled fibrils preserved the disorganization generated by unguided assembly of the network (Figure 2D). The images revealed internal fibril entanglement, branching, and, most critically, free fibrillar ends which were not observed in the fibers drawn from monomer-enriched droplet surface. This observation suggests that even cellular manipulation of an already polymerized network is unlikely to result in highly aligned fibrillar structures without further fibril modification. ${ }^{42}$

In a series of negative control experiments, we attempted to pull fibers from collagen solutions in which the ionic strength was reduced at low $\mathrm{pH}$ (nonpolymerizing conditions). Fibers could not be generated from these collagen solutions even after excessive exposure of $>10$ min to the dry nitrogen, confirming that intermolecular interactions, mediated by ions and mechanical strains, are required to produce the observed structure. We also examined the droplet surface at high resolution using quick freeze deep etch (QFDE) electron microscopy. Collagen droplets were rapidly frozen using a custom touch freezing technique, following $2.5 \mathrm{~min}$ of exposure to dry nitrogen (the time when a fiber could be reliably drawn from the ripened droplet). Figure 2E,F shows transmission electron microscopy (TEM) images of the replicated droplet surface. We found that a packed monomeric surface existed on the collagen droplets, with little evidence of surface polymerization/aggregation. 
The fibers produced by extensional strain were structurally unstable if they were left unloaded in solution for extended periods. This suggests that tensile mechanical forces not only generate the fibers but also constitute a stabilizing signal. Incubation of drawn telofibers at $37{ }^{\circ} \mathrm{C}$ in $1 \times \mathrm{PBS}$ for $48 \mathrm{~h}$ led to significant dissociation (see Figure S3). The fibril dissociation observed in the unloaded samples was prevented by application of a nominal tension to the fiber during incubation, as confirmed by TEM. The observed strain stabilization of the collagen fibrillar structure is consistent with strain-induced resistance of collagenous structures to both thermal ${ }^{31}$ and enzymatic ${ }^{32}$ degradation. It also supports the general concept that tension/strain may be a critical signal which extends the half-life of assembled biological polymers. ${ }^{43}$ Intermolecular stability can be enhanced by reducing the hydration of the collagen fibrils ${ }^{44,45}$ and is potentially equivalent to the effect of tensile strain. ${ }^{46}$ Thus, we probed the correlation between strain-induced stability and osmotically induced stability by incubating drawn fibers in $1.63 \%$ polyethylene glycol (PEG), chosen for its physiological match to the oncotic pressure of interstitial fluid. ${ }^{47}$ The treatment protected the fibers from dissociation as well as the application of mechanical tension.

Figure 3A-F shows the highly directional orientation and spontaneous development of tendon-like hierarchy induced in fibers by extensional strain. However, in spite of the strong axial organizing effect of the extensional flow, lateral fibrillar associations were often visible between single fibrils in images collected by scanning electron microscopy (Figure 3C). To more fully reproduce the conditions in vivo and provide a mechanism to control lateral fiber associations (a problem that is observed in collagen fibrillar structures when small leucinerich proteoglycans are absent ${ }^{48}$ ), fibers were drawn from a solution of collagen containing a physiological concentration of decorin (2\%). The addition of the decorin did not affect the ability to draw fibers, and an improvement in the discreteness of fibrils was readily observed (Figure 3F).

TEM images of fiber longitudinal sections revealed a heterogeneous shell and core structure, with an intermediate transitional zone, for both atelo-fibers (Figure 4) and telo-fibers (Figure 5). The external 5-10 $\mu \mathrm{m}$ thick "shell" comprised continuous, highly aligned fibrils in both atelo- and telo-fibers. The fibrils were discrete and evenly spaced in the atelo-fibers (Figure 4B,C) but were more densely packed in the shell of the telo-fibers (Figure 5A and Figure S4). Although some regions abruptly switched from shell structure to core (Figure 4D), the typical atelo-fiber transitional region comprised shorter, wavy fibrils (Figure 4E). The transitional region between the shell and core for telo-fibers was more extensive, beginning with short bands of fibrils (Figure 5A), generally at an angle to the long axis of the fiber. The shell to core transition progressively changed to a swirling morphology of short, poorly packed fibrils (Figure 5B). The core of both the atelo-fibers (Figure 4F) and telo-fibers (Figure 5C) had a similar appearance comprising discrete fibrils with isotropic orientation. Fibril banding periodicity for telo-fibers was calculated (Figure 5D) by isolating a region on an extracted fibril (shown between the brackets in Figure 5E) and performing a power spectral analysis. The average fibril periodicity for telo-fibers drawn from collagen solution containing the small leucine-rich proteoglycan decorin was $65.4 \pm 2.2 \mathrm{~nm}$. The periodicity closely matches with mammalian D-periodic banding. 
If a simple extensional strain analysis is applied to the measured geometry in the transition region from the droplet to the fiber, we estimate a maximum extensional strain rate of $0.5 \mathrm{~s}^{-1}$ (see Supporting Information). However, transitions in the ultrastructural arrangement of the internal fibrils from the shell of the fiber to the core clearly reflect the influence of complex fluid mechanics and the depth-dependent concentration gradient of collagen (see Supporting Information Figure S7). The swirling morphology and the angled patterning of fibrils between the shell and core of the fiber suggest variations in the extensional strain rate as a function of radial position and the presence of substantial shearing flows. Both have been neglected in the analytical solution. Direct examination of the detailed fluid mechanics in the necking region via fluorescent bead tracking revealed complex internal recirculation flows and shearing flows surrounded by a relatively well-behaved extensional flow (Figure 5F,G and Movie S3). Thus, we cannot assume a pure uniaxial extensional flow field, which is required to obtain an accurate analytical solution. Fortunately, it was possible to estimate the magnitude of the extensional strain rates directly from fluorescent bead tracks, which ranged from 0.1 to $0.7 \mathrm{~s}^{-1}$. Based on the concentration gradient data and the observed fluid mechanics, we propose four fluid mechanics/concentration regimes which may explain the resulting fibril morphology (Figure 5H): (I) the enriched collagen on the droplet surface experiences FIC as it transits through the necking region in the extensional strain region, forming highly aligned fibrillar structures; (II) in the next layer, less enriched collagen experiences more shearing strain, leading to organized but less aligned fibrillar structures; (III) there is a transition region where the shearing flow and the recirculating flow mix; and (IV) there is a recirculation zone where lower concentration collagen is incorporated into the fiber, which results in sparse, isotropic fibrillar organization. Our direct observation of the fluid mechanical behavior in the necking region was consistent with the resulting fibril structure within the drawn fiber.

\section{Discussion}

Flow-induced crystallization of mildly concentrated $(\sim 15 \mathrm{mg} / \mathrm{mL})$ type I collagen monomers into native-like, hierarchical, highly oriented fibers at very low extensional strain rates suggests a potentially powerful mechanism capable of driving the formation, growth, and continuity of load-bearing, collagenous tissue. The additional finding that mechanical strain stabilizes drawn fibers suggests that collagen possesses a physicochemical predilection to both accumulate and be retained in the path of tensile forces. While the results are important to engineers attempting to create organized collagenous structures de novo, they may also tell us something quite important about the evolution of structural control in modern metazoans. In primitive pre-Cambrian metazoans such as the sponge, collagen fibrils are deposited into the ECM rather randomly by wandering lophocytes, ${ }^{49}$ leading to structures poorly suited to effectively bear load. In contrast, post-Cambrian metazoans generate tissue comprising exquisitely organized arrays of collagen fibrils which persist over long distances and are generally aligned in the path of force. The transition from poor control to exquisite control of collagen structure occurred during a very short evolutionary window about 540 MYA. ${ }^{50}$ It is possible that adaptations which enabled FIC of collagen, in conjunction with improved cellular migration and organizational patterning, drastically improved the 
controllability of the ECM. However, the mechanisms of collagen structure formation, in general, even in modern metazoans, require further examination.

In spite of long-standing questions regarding tissue assembly, how large length scale, continuous structures can be generated by small length scale, discontinuous cells, using materials that possess submicrometer dimensions remains unclear. ${ }^{51}$ Even after 100 years of investigation into collagen assembly and transport by fibroblasts, ${ }^{52}$ neither the precise location of collagen fibril nucleation (inside, ${ }^{20}$ outside or on the cell surface ${ }^{22}$ ) nor the mechanisms that control fibril organization and growth have been definitively identified. It is clear, however, that the cells of the developing mesenchyme play a significant role guiding the initial assembly and transport of collagen to the ECM. ${ }^{19,53,54}$ In two of the most highly organized and investigated collagenous tissues, tendon and cornea, mesenchymal fibroblasts display specialized cell surface invaginations or "fibripositors" that associate intimately with newly forming collagen fibrils. ${ }^{19,20,55}$ Originally, fibripositors were thought to "vectorially discharge" collagen fibril segments into the extracellular space, ${ }^{56}$ where they spontaneously fuse end-to-end and laterally while aggregating to produce bundles of continuous fibrils. ${ }^{57}$

Although forces had long been considered potent epigenetic drivers of morphogenesis, ${ }^{58,59}$ early models of collagen fibril formation did not specifically incorporate mechanics as an important factor governing either collagen assembly or fibril fusion. In both the developing tendon and cornea, however, loss of mechanical tension during development has profound effects, resulting in the disintegration of tendon rudiments ${ }^{60}$ and in a drastic reduction in corneal growth. ${ }^{61,62}$ In addition, over the past decade, cell contractile proteins ${ }^{21,22}$ and matrix tension ${ }^{23}$ have been shown to be instrumental to the formation of tendon fibripositors and to the generation of collagen fibrils. ${ }^{22}$ The view of fibripositors has been expanded, and they are now thought to be traction-driven invaginations that apply tension directly to fibrils, drawing them toward the cell. ${ }^{22}$ In this expanded model, traction forces serve to align fibrils that already exist while polymerization occurs preferentially on the distal fibril tips which serve as nucleators. The role of mechanics as a director of collagen assembly was touched upon by Kadler and co-workers when they suggested that fibronectin or integrin-mediated acceleration of fibrillo-genesis through a conformational change in collagen is a "tantalizing possibility". ${ }^{63}$ In the cornea, 3D electron microscopy recently revealed assembling collagen fibrils colocalized along the axes of an orthogonal array of extended, actin-rich cell processes which resemble filopodia. ${ }^{64}$ These extensions are sites of $a 5 \beta 1$ integrin clustering, linking fibronectin to collagen fibrillogenesis. ${ }^{64}$ Thus, cell extensions appear to represent a second site of collagen assembly, far from the main cell body. These observations raise significant questions which are difficult to answer even with an expanded fibripositor/spontaneous fibril fusion model.

Our results provide a physical mechanism which explains how tension can directly drive initial fibrillogenesis and facilitate a rapid approach to fibril fusion/continuity. We here propose a model of early connective tissue development where mechanical strain initiates fibril formation, provides a mechanism to grow initial structures into the path of force, and ultimately establishes long-range connectivity. These mechanical signals extend the reach of the cells and act over longer length scales to instruct the efficient production, placement, growth, and connectivity of collagen fibrils. In our proposed model, following cell recruitment ${ }^{65}$ (Figure 6A), organization (Figure 6B), and ECM synthesis (Figure 6C), initial 
collagen fibril assembly is triggered by extensional strain. Regions of the cell membrane that contain contractile filaments (e.g., filopodia) retract inward, and the extensional strain is transferred to the collagen-rich solution surrounding the mesenchymal cells (Figure 6D). The extensional strain rate is maximized in the region of separation. The adjacent regions of high shear strain rate along the surfaces of the cell extension serve to prealign the collagen to enhance the effect of the extensional strain.

The physics of extensional strain-induced crystallization are highly relevant to developing biological systems which comprise high aspect ratio cells and fibrils embedded in viscous fluid. To form a fibril from solution, our data suggest that extensional strain rates between 0.1 and $0.7 \mathrm{~s}^{-1}$ are sufficient for collagen concentrations near $15 \mathrm{mg} / \mathrm{mL}(\sim 50 \mu \mathrm{M})$. The juxtacellular concentration of collagen during development is not known; however, the required concentration appears physiologically achievable. First, the rate of collagen synthesis during development ${ }^{19}$ can raise the concentration within $5 \mu \mathrm{m}$ of the cell surface to $\sim 50 \mu \mathrm{M}$ in $5 \mathrm{~h}$. Second, collagen can be exported directly at concentrations which are in the 10 s of $\mathrm{mg} / \mathrm{mL}$ range. ${ }^{66}$ Third, hyaluronic acid (HA), a powerful osmotic molecule ${ }^{67}$ and network-forming protein excluder, ${ }^{68}$ is present in significant quantities during the initiation of fibrillo-genesis. ${ }^{69,70}$ We believe that HA molecularly crowds newly exported collagen monomers against the cell, onto surface receptors such as $a 2 \beta 1$ integrins, and onto cryptic fibronectin receptors. ${ }^{71}$ Once collagen is crowded into position, application of extensional strain substantially lowers the energy barrier to nucleation and accelerates fibril crystallization in the path of force. The required extensional strain rate also appears physiologically achievable. Fibroblasts have demonstrated enormous capacity to contract collagen substrates ${ }^{72}$ and to reel in collagen fibrils in a "hand-over-hand" manner. ${ }^{73}$ In developing tissue, we propose that this behavior serves to generate extensional strains which initiate and stabilize fibrils in the direction of the tension. We assume that local pulling occurs continuously until the mechanical resistance of the collagen is sufficient to stall the molecular motors driving contraction. If we use the data of Kress et al., ${ }^{74}$ which puts the maximum speed of cell filopodial retraction at $600 \mathrm{~nm} / \mathrm{s}$, extensional strain rates in excess of $0.1 \mathrm{~s}^{-1}$ can be maintained for distances of $\sim 10 \mu \mathrm{m}$ (Figure S8a). Local cell contractions are thus capable of driving crystallization of short fibrils. Superimposed global tissue extensions (Figure 6E), generated by muscle contractions applied to the developing rudiment during initial collagen fibrillogenesis, can also drive fibril formation and promote fusion.

Schweitzer et al. ${ }^{65}$ notes that even when the tendon rudiment is mostly cellular, the cellular interactions alone are adequate for transmitting the forces generated from spontaneous movements observed in embryos.

Fusion of fibrils proceeds because the exposed ends of fibrils and cell processes serve as extensional strain rate amplifiers. From polymer physics, it is known that when composite viscous solutions containing stiff high aspect ratio objects aligned with the direction of force (e.g., cells, filopodia, and fibrils in a developing tissue) are extensionally strained, there is a strain rate amplifying effect between the tips of the objects ${ }^{75}$ (Figure S8b). The ratio of the extensional strain rate between the objects to the global extensional strain rate can be written as 


$$
\frac{\dot{\varepsilon}_{\text {gap }}}{\dot{\varepsilon}_{\text {global }}}=1+\frac{L}{W}
$$

where $\dot{\varepsilon}_{\text {gap }}$ is the strain rate between the objects, $\dot{\varepsilon}_{\text {global }}$ is the strain rate applied to the whole tissue rudiment, $\mathrm{L}$ is the length of the object, and $W$ is the spacing between the ends. One can easily see from this relationship that the interobject extensional strain rate is double that of the applied extensional strain rate when the objects are about as long as the spaces between them. As the objects lengthen and the spaces between them shorten, the relationship expresses its hyperbolic nature and $\dot{\varepsilon}_{\text {gap }}$ rises rapidly. Thus, in the developing tissue, flowinduced crystallization should rapidly fuse fibril ends separated by small gaps.

If the proposed mechanism for collagen fibrillogenesis is correct, then one would expect morphogenesis of connective tissues to begin with the large-scale production of short fibril segments that are aligned with extensional strain. Birk et al. ${ }^{76}$ showed that, during initial tendon fibrillogenesis, 60-80\% of newly formed collagen fibrils (14D in chick) averaged $10.3 \mu \mathrm{m}$ in length. As eq 1 shows, once fibril segments are formed, they will become new sites of fibrillogenesis by magnifying extensional strain, preferentially inducing new fibril formation between them. This too is consistent with observations that fibril segments exhibit end-to-end growth. ${ }^{77}$ As fibrillogenesis proceeds, our model predicts a rapid increase in the fibril length (as is observed ${ }^{78}$ ) preferentially in the path of tension, leading inevitably to stable continuity of the collagenous structure. While our model does not address the initial migration and organization of cells or deal with the development of tissue hierarchy, it does provide insight into how initial short fibrils can form preferentially in the path of force and predicts the observed rapid establishment of fibril continuity over long distances. A fibrilforming mechanism which is triggered by extensional strain will generate material wherever there are small gaps or weaknesses in a system of fibril segments, so long as there is sufficient soluble monomer present. In this way, the growing system of fibers would be spontaneously "self-healing" via fibril fusion.

This proposed model for tissue formation and growth requires that the cells control both the molecular and the mechanical environment, as well as their own patterning. In adult scar repair, these conditions normally fail to be met. First, the immune system is competent, adding an entire class of invasive cells and molecular factors to the local ECM chemical milieu, ${ }^{79}$ which alters the fibroblastic response. Second, the length scales are enormous relative to development, and the repairing cells are unable to exert full control over the wound geometry. Instead, the cells typically must work at the wound periphery, applying high local forces to contract the wound. ${ }^{80}$ The injury site alters both the chemical and the mechanical signals and also challenges the effort to molecularly crowd the space with collagen. The mechanism of wound repair and remodeling is likely to incorporate some form of strain-driven collagen assembly, perhaps direct molecular incorporation into loaded fibrils; however, the efficiency is likely much smaller. Early embryonic wound healing more closely resembles developmental tissue formation since the wound sizes are smaller, the immune system is incompetent, and the external factors are more controlled. 
There are substantial uncertainties associated with the proposed model. We are uncertain of the magnitude of the contraction speeds produced by developmental fibroblasts in the mesenchyme. They may be too low to induce initial fibrillogenesis of fibrils of the length observed. The existence of compartments in tendon development, which surround developing fibril bundles, could shield them from externally applied extensional strains, limiting the mechanism's effect. We do not know the actual concentration of collagen next to the cells nor can we say why it does not spontaneously assemble at $50 \mu \mathrm{M}$ as it would in vitro. However, given that there are approximately 50 molecules capable of associating with collagen, ${ }^{63}$ there is significant potential for modification of the minimum nucleation concentration. The observation that new fibrils form parallel to filopodia, ${ }^{64}$ observations noting that fibrils form along the long axis of fibroblasts, ${ }^{81}$ and reports of significant lateral fusion of fibril segments ${ }^{78}$ suggest that assembly may also be triggered in the high shear field next to moving structures. We have not directly investigated shear driven assembly in this paper, ${ }^{15}$ but we did see evidence of shear effects in our fibers between the shell and core (Figure 5D). Finally, it is possible that fibrillogenesis and growth of tissue could be accomplished in the absence of mechanically driven assembly through other mechanisms such as molecular crowding ${ }^{82}$ in conjunction with geometric confinement, contact guidance, and preferential assembly of collagen seeded by the fibril ends. ${ }^{57,77,78}$

\section{Conclusions}

In this study, we have demonstrated controlled assembly of highly aligned collagen fibrillar arrays via flow-induced crystallization at cell-achievable strain rates and at physiologically achievable collagen concentrations. The fibers produced by this process were stabilized when placed under tensile loads. The findings immediately support a model of tissue morphogenesis that combines flow-induced crystallization with the well-known ability of fibroblastic cells to generate substantial traction forces. ${ }^{72}$ The model is consistent with longstanding and more recent observations of connective tissue generation during development and directly addresses fibril progression from short fibril segments to tissue continuity. The finding that strain stabilizes fibrils may also shed light on the undesirable persistence of scars and fibrotic tissues. We conclude that extensional strain is a robust, long-range signal with the potential to initiate the formation of collagen arrays and then drive them to continuity.

\section{Methods \\ Collagen Preparation}

Two bovine dermal collagen sources were used in the creation of aligned collagen fibers. Pepsin-extracted collagen (5010-D, Advanced Biomatrix) and acetic-acid-extracted collagen (5026-D, Advanced Biomatrix) were used with a starting concentration of $5.8 \mathrm{mg} / \mathrm{mL}$ suspended in $0.01 \mathrm{M} \mathrm{HCl}$. The pepsin-extracted collagen was neutralized using an 8:1:1 ratio of collagen, 10× PBS (BP399-1, Fisher Scientific), and 0.1 M NaOH (12419-0010, Fisher Scientific), respectively. This resulted in a $\mathrm{pH}$ of 7.3. For the experiments using decorin (D8428-.5MG, Sigma-Aldrich), a ratio of 2\% decorin to collagen molar ratio was used. The $0.5 \mathrm{mg}$ of lyophilized decorin was first reconstituted with $1 \mathrm{~mL}$ of deionized 
water. To prevent any spontaneous polymerization while working with acetic-acid-extracted collagen, the neutralization ratio was altered such that the amount of $0.1 \mathrm{M} \mathrm{NaOH}$ was increased to $115 \%$. Consequently, this resulted in a $\mathrm{pH}$ of 7.7. However, no fibrils were detected via differential interference contrast microscopy for twice as long as the experimental time.

\section{Fiber Drawing Setup}

The collagen fiber drawing setup, shown in Figure S1, was located on a Nikon TE-2000E microscope for live imaging and visual feedback. A custom nitrogen diffusing chamber was designed to provide humidity control and enhance the evaporation rate through convection. A nitrogen tank, regulated to 0.022 psi by a digital pressure controller (PCDAS-1PSIG-D/5P, 5IN, Alicat Scientific), supplied the chamber's one primary inlet that then branched off to six nozzles to provide a constant flow of dry nitrogen gas. A $125 \mu \mathrm{L}$ droplet of neutralized collagen solution sat on top of an $8 \mathrm{~mm}$ glass coverslip, which rested on top of a $40 \mathrm{~mm}$ glass coverslip. The $8 \mathrm{~mm}$ coverslip was essential for pinning the boundary of the droplet, resulting in a repeatable initial geometry and evaporation profile. The $40 \mathrm{~mm}$ coverslip was used to provide the optical access to the inverted microscope. A glass microneedle, held by a capillary holder and moved by an electronic micromanipulator (TransferMan NK 2, Eppendorf), was used as the probe to draw the collagen fiber. The chamber lid was mounted on the condenser lens of the microscope to isolate the chamber volume from the local humidity in the room.

\section{Fiber Drawing Protocol}

Prior to making the neutralized collagen solution, $5 \mathrm{~min}$ with the nitrogen gas flowing was allotted to equilibrate the humidity level within the chamber to $<5 \%$ humidity. The collagen solution was then made by first combining the $\mathrm{NaOH}$ and $\mathrm{PBS}$ components and last adding the collagen component. This reduced the initial $\mathrm{pH}$ shock and prevented any instantaneous precipitation. The solution was then pipetted in and out (carefully avoiding introduction of air bubbles) $\geq 15$ times to yield a homogeneous, neutralized solution. Next, $125 \mu \mathrm{L}$ was pipetted onto the $8 \mathrm{~mm}$ glass coverslip, again avoiding the addition of any air bubbles. The microneedle was held just above the apex of the droplet, and the system was untouched for $150 \mathrm{~s}$. After the allotted time, the needle was lowered into the droplet $25-50 \mu \mathrm{m}$, and a prewritten program in NIS Elements AR v4.13 raised the microneedle (Table 1).

The initial wait step was required to consistently pull a fiber of appreciable length (>10 $\mathrm{mm}$ ), and the slower starting velocity was designed to initiate the formation of an attachment that could withstand the release of the meniscus. The $90 \mu \mathrm{m} / \mathrm{s}$ velocity aided in smoothly transitioning to a $240 \mu \mathrm{m} / \mathrm{s}$ velocity without breaking the fiber. The $240 \mu \mathrm{m} / \mathrm{s}$ velocity best paired with the speed of the evaporative surface enrichment, such that over the length of the fiber there was little visible thinning or thickening. The final step was included to ramp down the velocity as the source of enriched collagen was also equivalently depleted from the droplet. Once the fiber pull had completed, the fiber was given 1 min to dry before being stored for later analysis. 


\section{Calculating the Extensional Strain Rate}

The extensional strain rate was calculated by recording the motion of fluorescent, $1.9 \mu \mathrm{m}$ microspheres (G0200, Thermo Scientific) during the fiber drawing process (Movie S3) at 7 frames per second. The position and time stamp of each video frame was used to calculate the $2 \mathrm{D}$ velocity magnitude, assumed to be the velocity along the streamline, of an individual bead moving through the necking region of the droplet. The extensional strain rate was then determined as the change in the $2 \mathrm{D}$ velocity magnitude divided by the distance the bead moved between each frame. Although the streamline along the necking region was a curved path, the incremental analysis allowed the distance to be approximated as a straight line with minimal error.

\section{Touch Freezing and Quick Freeze Deep Etch Sample Preparation}

Bothe atelo- and telo-collagen droplets were subjected to the fiber drawing protocol and were preserved just prior to actually drawing the fiber. The preservation process included mounting the droplet and coverslip on a linear motor (stator \#0150-1254 and slider \#0150-1524, LinMot), followed by a rapid approach toward a liquid-nitrogen-cooled copper mirror. The custom touch ${ }^{83}$ freezer brought the sample to $5 \mathrm{~mm}$ above the mirror surface at $2.5 \mathrm{~m} / \mathrm{s}$ to minimize excessive heat transfer, followed by a final approach at $2 \mathrm{~cm} / \mathrm{s}$ to minimize the mechanical distortion. The frozen droplets were stored in liquid nitrogen until they were transferred to the Freeze Etch device.

The Freeze Etch device was a modified Cressington freeze fracture system (CFE-40, Cressington Scientific Instruments). The droplets were etched inside the CFE-40 for $1 \mathrm{~h}$ at $-100{ }^{\circ} \mathrm{C}$ and $10^{-6}$ Torr to remove the vitrified ice from the surface of the droplet. Next, the QFDE temperature was reduced to $-130{ }^{\circ} \mathrm{C}$ to minimize the grain size $(1.5-2.5 \mathrm{~nm})$ when depositing both the platinum ( $\sim 4 \mathrm{~nm}$ thick) and carbon ( $\sim 10 \mathrm{~nm}$ thick) layers. The platinum/ carbon replica was then isolated from the collagen droplet via a digestion in $25 \%$ bleach. After $1 \mathrm{~h}$ in bleach, the replica was transferred to deionized water for $1 \mathrm{~h}$. Lastly, the replicas were picked up on 600 mesh copper grids and imaged using a JEOL JEM 1010 transmission electron microscope (JEOL, Japan).

\section{Atelo-Fiber Preparation for Transmission Electron Microscopy}

Atelo-fibers were prepared for transmission electron microscopy by incubating for $48 \mathrm{~h}$ at $37^{\circ} \mathrm{C}$ in PBS under nominal strain on the microscope (Figure 4) to observe if any visual changes were occurring to the structure of the fibers. The application of strain was used to maintain the fiber within a narrow focal plane for long-term imaging. No visual changes were observed during the incubation, so the fibers were then fixed with a recipe of $2.5 \%$ glutaraldehyde and $2.5 \%$ paraformaldehyde in a $0.1 \mathrm{M}$ sodium cacodylate buffer for $1 \mathrm{~h}$ at room temperature, followed by three washes, postfixation with $1 \%$ osmium tetroxide in 0.1 $\mathrm{M}$ sodium cacodylate buffer for $1 \mathrm{~h}$, three washes, and a graded series of ethanol exchanges. Fibers were embedded in resin and then longitudinally thin sectioned on a microtome (Ultracut E, Reichert) using a diamond knife. The $\sim 90 \mathrm{~nm}$ thin sections were plated on TEM grids and stained with 5\% uranyl acetate and 5\% Reynolds lead citrate. The sections were viewed on the JEOL JEM 1010 TEM, and images were digitally captured on a CCD camera system (AMT XR-41B, Advanced Microscopy Techniques Inc.). 


\section{Telo-Fiber Preparation for Scanning Electron Microscopy}

Telo-fibers were prepared for scanning electron microscopy (SEM) by incubating the fibers for $48 \mathrm{~h}$ at $37^{\circ} \mathrm{C}$ in PBS in an unloaded, unattached manner. This allowed for parallel processing of the samples but resulted in significant dissociation of the fibers (Figure S3). When the unloaded incubation was repeated for atelo-fibers, the fibers equivalently dissociated, and it was realized that stabilization (via strain) was essential for non-crosslinked fiber persistence at physiological temperature. To maintain a parallel processing approach, polyethylene glycol ( $\mathrm{MW}=20 \mathrm{kDa}$ ) was used at physiological concentration ${ }^{47}$ for its osmotic stabilizing effects ${ }^{46}$ (Figure $3 \mathrm{~A}-\mathrm{C}$ ), as a substitution to strain stabilization. Telofibers were also processed on the microscope with strain instead of PEG as a control, but no observable differences were recognized in the fiber morphology.

A second set of telo-fibers were prepared for SEM by including $2 \%$ decorin in the fiberpulling solution and then incubating the unloaded fibers for $48 \mathrm{~h}$ at $37^{\circ} \mathrm{C}$ in PBS containing $1.63 \%$ PEG and 2\% decorin (Figure 3D-F). After the incubation period, both sets of fibers were then fixed with a recipe of $2.5 \%$ glutaraldehyde and $2.5 \%$ paraformaldehyde in a $0.1 \mathrm{M}$ sodium cacodylate buffer for $1 \mathrm{~h}$ at room temperature, followed by three washes and a graded series of ethanol exchanges. The fibers were sputter coated with a carbon-platinum coating and SEM imaged (S-4800, Hitachi, Japan) for examination.

\section{Telo-Fiber Preparation for Transmission Electron Microscopy}

Telo-fibers were drawn with $2 \%$ decorin in the solution and prepared for TEM by incubating in an unloaded configuration for $48 \mathrm{~h}$ at $37^{\circ} \mathrm{C}$ in PBS with 1.63\% PEG and 2\% decorin (Figure 5). The fixation, sectioning, and imaging protocol used for the TEM of atelo-fibers was repeated for telo-fibers.

\section{Fiber Dissociation}

Incubation of the fibers at $37^{\circ} \mathrm{C}$, in the absence of strain, resulted in the progressive dissociation of the fibrils. Telo-fibers were evaluated by TEM imaging at two time points: 1 and $48 \mathrm{~h}$. After $1 \mathrm{~h}$, the most peripheral fibrils began separating from the fiber (Figure S3a). After $48 \mathrm{~h}$, the entire shell had nearly completely dissociated and the transitional region had increased empty space between bands of fibrils (Figure S3b). The observed changes were in stark contrast to fibers that were held with nominal strain or in a hypertonic solution during the incubation period. Strain and osmotic pressure appear to confer intermolecular stability that is required for the persistence of the collagen fibers in the absence of covalent crosslinks.

\section{Fibril Isolation}

Single fibrils from telo-fibers were isolated for TEM imaging by mechanically disrupting the fiber with a glass, microtissue grinder (885470-0000, Kimble Chase). A $10 \mathrm{~mm}$ central section of the fiber was suspended in the glass vial with $100 \mu \mathrm{L}$ of $1 \times$ PBS. The glass probe was used to disrupt the fiber until the solution turned opaque and no fiber fragments were visible. Twenty microliter aliquots were transferred to Formvar-coated TEM grids (01701-F, Ted Pella) for subsequent staining with $1.5 \%$ uranyl acetate and imaging. The grids were 
previously treated with $1 \%$ alcian blue to provide a more hydrophilic surface for the fibrils to adhere.

\section{Fibril Periodicity Analysis}

The fibril banding periodicity was estimated from the power spectra of TEM images of individual fibrils. Acceptable images were those where at least $500 \mathrm{~nm}$ of banded fibrils was clearly visible. First, each grayscale image was oriented such that the long axis of one fibril was horizontal and cropped to the largest straight region of banded fibril within the image (Figure S2a). Next, the grayscale value of pixels within each column in the cropped image was averaged (Figure S2b), and the 1D spatial signal was converted into its frequency domain using discrete Fourier transform. Then, the squared complex modulus of the frequency domain was computed to obtain the power spectral density plot (Figure S2c). In this plot, the largest peak falling between 60 and $80 \mathrm{~nm}$ was recognized as the banding periodicity. All image analysis and processing were performed in MATLAB (R2014a, MathWorks).

\section{Supplementary Material}

Refer to Web version on PubMed Central for supplementary material.

\section{Acknowledgments}

The senior author thanks James S. Ruberti for over a decade of insightful discussions about collagen autonomy. The authors thank Dr. Barbara Brodsky at Tufts University for her collagen expertise and invaluable advice. The authors thank Dr. Pirouz Kavehpour for his rheology expertise and scientific discussions. The first author received support as a Charles Stark Draper Laboratory Fellow. The study was supported by R01 NEI EY015500.

\section{References}

1. Garrone R. Collagen, a Common Thread in Extracellular Matrix Evolution. Proc Indian Acad Sci (Chem Sci). 1999; 111:51-56.

2. King N, Westbrook MJ, Young SL, Kuo A, Abedin M, Chapman J, Fairclough S, Hellsten U, Isogai Y, Letunic I, Marr M, Pincus D, Putnam N, Rokas A, Wright KJ, Zuzow R, Dirks W, Good M, Goodstein D, Lemons D, et al. The Genome of the Choanoflagellate Monosiga Brevicollis and the Origin of Metazoans. Nature. 2008; 451:783-788. [PubMed: 18273011]

3. Frosler J, Leadbeater BS. Role of the Cytoskeleton in Choanoflagellate Lorica Assembly. J Eukaryotic Microbiol. 2009; 56:167-173.

4. Boot-Handford RP, Tuckwell DS. Fibrillar Collagen: The Key to Vertebrate Evolution? A Tale of Molecular Incest. BioEssays. 2003; 25:142-151. [PubMed: 12539240]

5. Gross J, Highberger J, Schmitt F. Some Factors Involved in the Fibrogenesis of Collagen in Vitro. Exp Biol Med. 1952; 80:462.

6. Katz JN. Lumbar Disc Disorders and Low-Back Pain:Socioeconomic Factors and Consequences. J Bone Joint Surg Am. 2006; 88:21-24. [PubMed: 16595438]

7. Nagda SH, Altobelli GG, Bowdry KA, Brewster CE, Lombardo SJ. Cost Analysis of Outpatient Anterior Cruciate Ligament Reconstruction: Autograft Versus Allograft. Clin Orthop Relat Res. 2010; 468:1418-1422. [PubMed: 20020337]

8. Lyman S, Koulouvaris P, Sherman S, Do H, Mandl LA, Marx RG. Epidemiology of Anterior Cruciate Ligament Reconstruction: Trends, Readmissions, and Subsequent Knee Surgery. J Bone Joint Surg Am. 2009; 91:2321-2328. [PubMed: 19797565]

9. Arden N, Nevitt MC. Osteoarthritis: Epidemiology. Best Pract Res Clin Rheumatol. 2006; 20:3-25. [PubMed: 16483904] 
10. Bahr GF. The Reconstitution of Collagen Fibrils as Revealed by Electron Microscopy. Exp Cell Res. 1950; 1:603-606.

11. Lee P, Lin R, Moon J, Lee LP. Microfluidic Alignment of Collagen Fibers for in Vitro Cell Culture. Biomed Microdevices. 2006; 8:35-41. [PubMed: 16491329]

12. Cheng X, Gurkan UA, Dehen CJ, Tate MP, Hillhouse HW, Simpson GJ, Akkus O. An Electrochemical Fabrication Process for the Assembly of Anisotropically Oriented Collagen Bundles. Biomaterials. 2008; 29:3278-3288. [PubMed: 18472155]

13. Matthews JA, Wnek GE, Simpson DG, Bowlin GL. Electrospinning of Collagen Nanofibers. Biomacromolecules. 2002; 3:232-238. [PubMed: 11888306]

14. Paten JA, Tilburey GE, Molloy EA, Zareian R, Trainor CV, Ruberti JW. Utility of an OpticallyBased, Micromechanical System for Printing Collagen Fibers. Biomaterials. 2013; 34:2577-2587. [PubMed: 23352045]

15. Saeidi N, Sander EA, Ruberti JW. Dynamic Shear-Influenced Collagen Self-Assembly. Biomaterials. 2009; 30:6581-6592. [PubMed: 19765820]

16. Martin R, Farjanel J, Eichenberger D, Colige A, Kessler E, Hulmes DJ, Giraud-Guille MM. Liquid Crystalline Ordering of Procollagen as a Determinant of Three-Dimensional Extracellular Matrix Architecture. J Mol Biol. 2000; 301:11-17. [PubMed: 10926488]

17. Torbet J, Ronziere MC. Magnetic Alignment of Collagen During Self-Assembly. Biochem J. 1984; 219:1057-1059. [PubMed: 6743242]

18. Belamie E, Mosser G, Gobeaux F, Giraud-Guille MM. Possible Transient Liquid Crystal Phase During the Laying out of Connective Tissues: Alpha-Chitin and Collagen as Models. J Phys : Condens Matter. 2006; 18:115-129.

19. Birk DE, Trelstad RL. Extracellular Compartments in Matrix Morphogenesis: Collagen Fibril, Bundle, and Lamellar Formation by Corneal Fibroblasts. J Cell Biol. 1984; 99:2024-2033. [PubMed: 6542105]

20. Canty EG, Lu Y, Meadows RS, Shaw MK, Holmes DF, Kadler KE. Coalignment of Plasma Membrane Channels and Protrusions (Fibripositors) Specifies the Parallelism of Tendon. J Cell Biol. 2004; 165:553-563. [PubMed: 15159420]

21. Canty EG, Starborg T, Lu Y, Humphries SM, Holmes DF, Meadows RS, Huffman A, O'Toole ET, Kadler KE. Actin Filaments Are Required for Fibripositor-Mediated Collagen Fibril Alignment in Tendon. J Biol Chem. 2006; 281:38592-38598. [PubMed: 17020878]

22. Kalson NS, Starborg T, Lu Y, Mironov A, Humphries SM, Holmes DF, Kadler KE. Nonmuscle Myosin Ii Powered Transport of Newly Formed Collagen Fibrils at the Plasma Membrane. Proc Natl Acad Sci U S A. 2013; 110:E4743-4752. [PubMed: 24248360]

23. Kapacee Z, Richardson SH, Lu Y, Starborg T, Holmes DF, Baar K, Kadler KE. Tension Is Required for Fibripositor Formation. Matrix Biol. 2008; 27:371-375. [PubMed: 18262777]

24. Bhole AP, Flynn BP, Liles M, Saeidi N, Dimarzio CA, Ruberti JW. Mechanical Strain Enhances Survivability of Collagen Micronetworks in the Presence of Collagenase: Implications for LoadBearing Matrix Growth and Stability. Philos Trans R Soc, A. 2009; 367:3339-3362.

25. Changeux JP. 50th Anniversary of the Word “Allosteric”. Protein Sci. 2011; 20:1119-1124. [PubMed: 21574197]

26. Motlagh HN, Wrabl JO, Li J, Hilser VJ. The Ensemble Nature of Allostery. Nature. 2014; 508:331-339. [PubMed: 24740064]

27. Seifert C, Grater F. Protein Mechanics: How Force Regulates Molecular Function. Biochim Biophys Acta, Gen Subj. 2013; 1830:4762-4768.

28. Ejim OS, Blunn GW, Brown RA. Production of Artificial-Orientated Mats and Strands from Plasma Fibronectin: A Morphological Study. Biomaterials. 1993; 14:743-748. [PubMed: 8218723]

29. Bucay I, O'Brien ET 3rd, Wulfe SD, Superfine R, Wolberg AS, Falvo MR, Hudson NE. Physical Determinants of Fibrinolysis in Single Fibrin Fibers. PLoS One. 2015; 10:e0116350. [PubMed: 25714359]

30. Jesudason R, Black L, Majumdar A, Stone P, Suki B. Differential Effects of Static and Cyclic Stretching During Elastase Digestion on the Mechanical Properties of Extracellular Matrices. J Appl Physiol. 2007; 103:803-811. [PubMed: 17540839] 
31. Lennox F. Shrinkage of Collagen. Biochim Biophys Acta. 1949; 3:170-187.

32. Flynn BP, Bhole AP, Saeidi N, Liles M, Dimarzio CA, Ruberti JW. Mechanical Strain Stabilizes Reconstituted Collagen Fibrils against Enzymatic Degradation by Mammalian Collagenase Matrix Metalloproteinase 8 (Mmp-8). PLoS One. 2010; 5:e12337. [PubMed: 20808784]

33. Breslauer DN, Lee LP, Muller SJ. Simulation of Flow in the Silk Gland. Biomacromolecules. 2009; 10:49-57. [PubMed: 19053289]

34. Rao I, Rajagopal K. A Study of Strain-Induced Crystallization of Polymers. Int J Solids Struct. 2001; 38:1149-1167.

35. Keller, A., Kolnaar, HWH. Flow-Induced Orientation and Structure FormationWiley; New York: 1997

36. Mackley MR, Keller A. Flow Induced Polymer-Chain Extension and Its Relation to Fibrous Crystallization. Philos Trans R Soc, A. 1975; 278:29-66.

37. Nestler FHM, Hvidt S, Ferry JD, Veis A. Flexibility of Collagen Determined from Dilute Solution Viscoelastic Measurements. Biopolymers. 1983; 22:1747-1758. [PubMed: 6882874]

38. Yamakawa H. Viscoelastic Properties of Straight Cylindrical Macromolecules in Dilute Solution. Macromolecules. 1975; 8:339-342.

39. Gobeaux F, Belamie E, Mosser G, Davidson P, Asnacios S. Power Law Rheology and StrainInduced Yielding in Acidic Solutions of Type I-Collagen. Soft Matter. 2010; 6:3769-3777.

40. Everaers R, Sukumaran SK, Grest GS, Svaneborg C, Sivasubramanian A, Kremer K. Rheology and Microscopic Topology of Entangled Polymeric Liquids. Science. 2004; 303:823-826. [PubMed: 14764875]

41. Kuznetsova N, Leikin S. Does the Triple Helical Domain of Type I Collagen Encode Molecular Recognition and Fiber Assembly While Telopeptides Serve as Catalytic Domains? Effect of Proteolytic Cleavage on Fibrillogenesis and on Collagen-Collagen Interaction in Fibers. J Biol Chem. 1999; 274:36083-36088. [PubMed: 10593890]

42. Wang H, Abhilash A, Chen CS, Wells RG, Shenoy VB. Long-Range Force Transmission in Fibrous Matrices Enabled by Tension-Driven Alignment of Fibers. Biophys J. 2014; 107:25922603. [PubMed: 25468338]

43. Dingal PDP, Discher DE. Systems Mechanobiology: Tension-Inhibited Protein Turnover Is Sufficient to Physically Control Gene Circuits. Biophys J. 2014; 107:2734-2743. [PubMed: 25468352]

44. Wenger MP, Bozec L, Horton MA, Mesquida P. Mechanical Properties of Collagen Fibrils. Biophys J. 2007; 93:1255-1263. [PubMed: 17526569]

45. van der Rijt JA, van der Werf KO, Bennink ML, Dijkstra PJ, Feijen J. Micromechanical Testing of Individual Collagen Fibrils. Macromol Biosci. 2006; 6:697-702. [PubMed: 16967482]

46. Masic A, Bertinetti L, Schuetz R, Chang SW, Metzger TH, Buehler MJ, Fratzl P. Osmotic Pressure Induced Tensile Forces in Tendon Collagen. Nat Commun. 2015; 6:5942. [PubMed: 25608644]

47. Sverdlik A, Lanir Y. Time-Dependent Mechanical Behavior of Sheep Digital Tendons, Including the Effects of Preconditioning. J Biomech Eng. 2002; 124:78-84. [PubMed: 11871608]

48. Raspanti M, Viola M, Sonaggere M, Tira ME, Tenni R. Collagen Fibril Structure Is Affected by Collagen Concentration and Decorin. Biomacromolecules. 2007; 8:2087-2091. [PubMed: 17530890]

49. Garrone, R. Phylogenesis of Connective Tissue: Morphological Aspects and Biosynthesis of Sponge Intercellular Matrix Vol. 5. S Karger Publishers; 1978

50. Marshall CR. Explaining the Cambrian “Explosion” of Animals. Annu Rev Earth Planet Sci. 2006; 34:30.

51. Kadler KE. The Needle in the Ecm Haystack. Nat Rev Mol Cell Biol. 2014; 15:769.

52. Mall FP. On the Development of Connective Tissues from the Connective Tissue Syncytium. Am J Anat. 1902; 1:329-365.

53. Trelstad RL, Birk DE. The Fibroblast in Morphogenesis and Fibrosis: Cell Topography and Surface-Related Functions. Ciba Found Symp. 2008; 114:4-19.

54. Kadler KE, Holmes DF, Trotter JA, Chapman JA. Collagen Fibril Formation. Biochem J. 1996; 316:1-11. [PubMed: 8645190] 
55. Birk DE, Trelstad RL. Fibroblasts Create Compartments in the Extracellular Space Where Collagen Polymerizes into Fibrils and Fibrils Associate into Bundles. Ann N Y Acad Sci. 1985; 460:258-266. [PubMed: 3868950]

56. Birk, DE., Silver, FH., Trelstad, RL. Matrix Assembly In Cell Biology of the Extracellular MatrixHay, ED., editorSpringer; New York: 1991

57. Birk DE, Nurminskaya MV, Zycband EI. Collagen Fibrillogenesis in Situ: Fibril Segments Undergo Post-Depositional Modifications Resulting in Linear and Lateral Growth During Matrix Development. Dev Dyn. 1995; 202:229-243. [PubMed: 7780173]

58. Wolff, J. Das Gesetz der Transformation der Knochen by P Maquet and R Furlongi (translated from the 1892 original)Springer Verlag; Berlin: 1986The Law of Bone Remodeling.

59. Thompson, DW. On Growth and FormCambridge University Press; Cambridge, UK: 1917

60. Kieny M, Chevallier A. Autonomy of Tendon Development in the Embryonic Chick Wing. J Embryol Exp Morphol. 1979; 49:153-165. [PubMed: 448266]

61. Bee JA. Intraocular Pressure-Dependent and -Independent Phases of Growth of the Embryonic Chick Eye and Cornea. Invest Ophthalmol Vis Sci. 1991; 32:2483-2491. [PubMed: 1869402]

62. Coulombre AJ. The Role of Intraocular Pressure in the Development of the Chick Eye. Ii. Control of Corneal Size. AMA Arch Ophthalmol. 1957; 57:250-253. [PubMed: 13393895]

63. Kadler KE, Hill A, Canty-Laird EG. Collagen Fibrillogenesis: Fibronectin, Integrins, and Minor Collagens as Organizers and Nucleators. Curr Opin Cell Biol. 2008; 20:495-501. [PubMed: 18640274]

64. Young RD, Knupp C, Pinali C, Png KM, Ralphs JR, Bushby AJ, Starborg T, Kadler KE, Quantock AJ. Three-Dimensional Aspects of Matrix Assembly by Cells in the Developing Cornea. Proc Natl Acad Sci U S A. 2014; 111:687-692. [PubMed: 24385584]

65. Schweitzer R, Zelzer E, Volk T. Connecting Muscles to Tendons: Tendons and Musculoskeletal Development in Flies and Vertebrates. Development. 2010; 137:2807-2817. [PubMed: 20699295]

66. Hulmes D, Bruns RR, Gross J. On the State of Aggregation of Newly Secreted Procollagen. Proc Natl Acad Sci U S A. 1983; 80:388-392. [PubMed: 6572898]

67. Laurent TC, Ogston AG. The Interaction between Polysaccharides and Other Macromolecules. 4. The Osmotic Pressure of Mixtures of Serum Albumin and Hyaluronic Acid. Biochem J. 1963; 89:249-253. [PubMed: 14084608]

68. Scott JE, Cummings C, Brass A, Chen Y. Secondary and Tertiary Structures of Hyaluronan in Aqueous Solution, Investigated by Rotary Shadowing-Electron Microscopy and Computer Simulation. Hyaluronan Is a Very Efficient Network-Forming Polymer. Biochem J. 1991; 274:699-705. [PubMed: 2012600]

69. Scott JE, Hughes EW. Proteoglycan-Collagen Relationships in Developing Chick and Bovine Tendons. Influence of the Physiological Environment. Connect Tissue Res. 1986; 14:267-278. [PubMed: 2938882]

70. Toole BP, Trelstad RL. Hyaluronate Production and Removal During Corneal Development in the Chick. Dev Biol. 1971; 26:28-35. [PubMed: 5111769]

71. Legant WR, Chen CS, Vogel V. Force-Induced Fibronectin Assembly and Matrix Remodeling in a 3d Microtissue Model of Tissue Morphogenesis. Integr Biol (Camb). 2012; 4:1164-1174. [PubMed: 22961409]

72. Harris AK, Stopak D, Wild P. Fibroblast Traction as a Mechanism for Collagen Morphogenesis. Nature. 1981; 290:249-251. [PubMed: 7207616]

73. Meshel AS, Wei Q, Adelstein RS, Sheetz MP. Basic Mechanism of Three-Dimensional Collagen Fibre Transport by Fibroblasts. Nat Cell Biol. 2005; 7:157-164. [PubMed: 15654332]

74. Kress H, Stelzer EH, Holzer D, Buss F, Griffiths G, Rohrbach A. Filopodia Act as Phagocytic Tentacles and Pull with Discrete Steps and a Load-Dependent Velocity. Proc Natl Acad Sci U S A. 2007; 104:11633-11638. [PubMed: 17620618]

75. Ahamadi M, Harlen O. A Lagrangian Finite Element Method for Simulation of a Suspension under Planar Extensional Flow. J Comput Phys. 2008; 227:7543-7560.

76. Birk DE, Zycband EI, Winkelmann DA, Trelstad RL. Collagen Fibrillogenesis in Situ: Fibril Segments Are Intermediates in Matrix Assembly. Proc Natl Acad Sci U S A. 1989; 86:4549-4553. [PubMed: 2734306] 
77. Kadler KE, Holmes DF, Graham H, Starborg T. Tip-Mediated Fusion Involving Unipolar Collagen Fibrils Accounts for Rapid Fibril Elongation, the Occurrence of Fibrillar Branched Networks in Skin and the Paucity of Collagen Fibril Ends in Vertebrates. Matrix Biol. 2000; 19:359-365. [PubMed: 10963997]

78. Birk DE, Zycband EI, Woodruff S, Winkelmann DA, Trelstad RL. Collagen Fibrillogenesis in Situ: Fibril Segments Become Long Fibrils as the Developing Tendon Matures. Dev Dyn. 1997; 208:291-298. [PubMed: 9056634]

79. Nourissat G, Berenbaum F, Duprez D. Tendon Injury: From Biology to Tendon Repair. Nat Rev Rheumatol. 2015; 11:223-233. [PubMed: 25734975]

80. Brugues A, Anon E, Conte V, Veldhuis JH, Gupta M, Colombelli J, Munoz JJ, Brodland GW, Ladoux B, Trepat X. Forces Driving Epithelial Wound Healing. Nat Phys. 2014; 10:683-690. [PubMed: 27340423]

81. Saeidi N, Guo X, Hutcheon AE, Sander EA, Bale SS, Melotti SA, Zieske JD, Trinkaus-Randall V, Ruberti JW. Disorganized Collagen Scaffold Interferes with Fibroblast Mediated Deposition of Organized Extracellular Matrix in Vitro. Biotechnol Bioeng. 2012; 109:2683-2698. [PubMed: 22528405]

82. Saeidi N, Karmelek KP, Paten JA, Zareian R, DiMasi E, Ruberti JW. Molecular Crowding of Collagen: A Pathway to Produce Highly-Organized Collagenous Structures. Biomaterials. 2012; 33:7366-7374. [PubMed: 22846420]

83. Ritzakis, N. Masters ThesisNortheastern University; 2011Method and Evaluation for Minimization of Mechanical Effects from Impact Velocity for the Optimization of Freezing Quality of Metal Mirror Impact Freezers. 


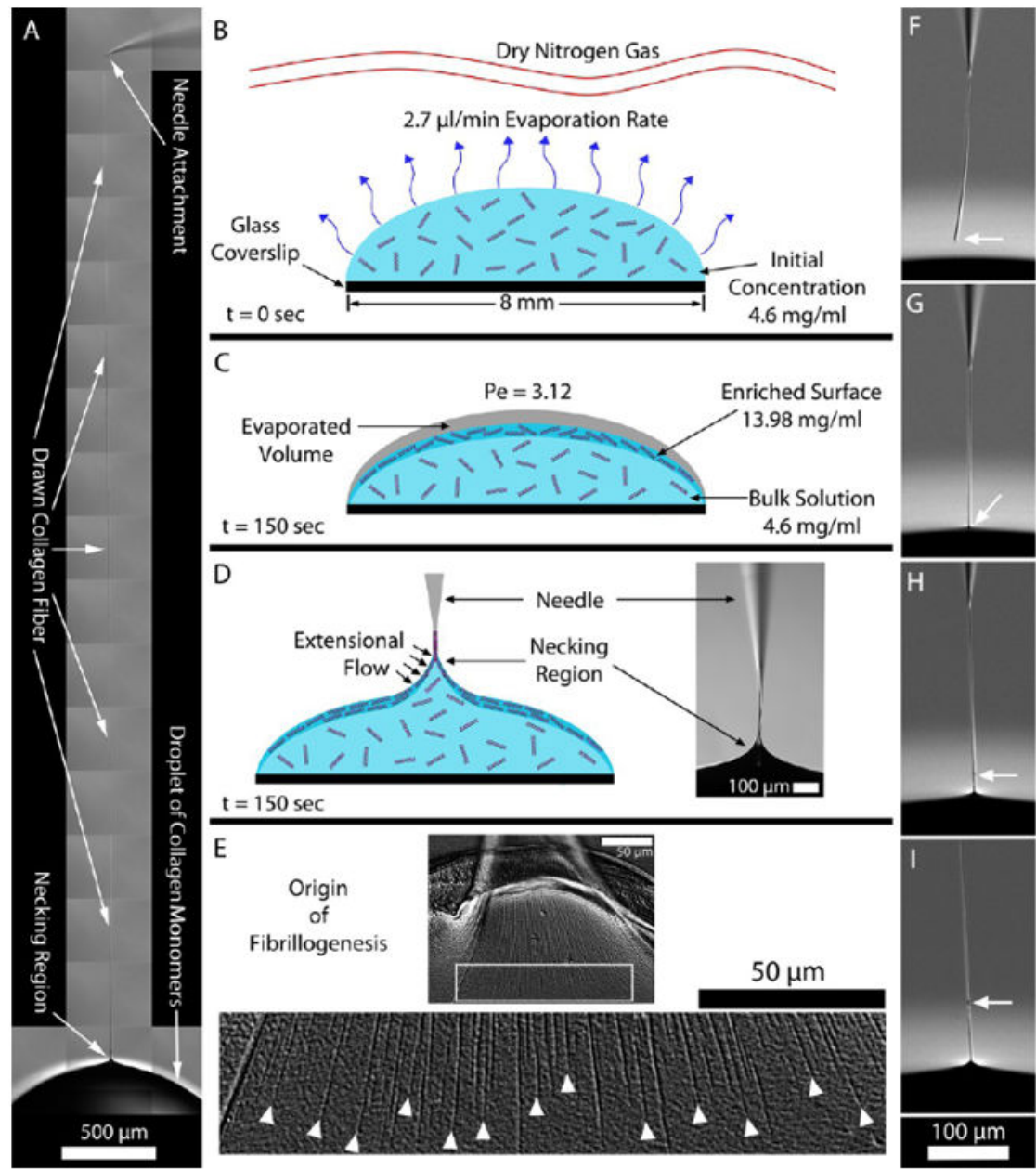

Figure 1.

(A) Fiber drawn from a droplet of collagen solution demonstrates the high aspect ratio fibers that can be created. (B-D) Cross-sectional schematics of the collagen droplet and surrounding environment at different time points. (B) Dry nitrogen gas was passed above the droplet to facilitate evaporation from the droplet surface. (C) After $150 \mathrm{~s}$, an enriched monomeric surface was produced as a result of the water molecules exiting the droplet faster than the collagen molecules could diffuse into the bulk (calculated Peclet number $(\mathrm{Pe})$ was 3.12). At this point, a collagen fiber could be reliably drawn from the droplet. (D) Glass microneedle was used to pierce the droplet surface and then drawn back outward. As the meniscus of the droplet drained from the microneedle, a collagen fiber persisted. (E) Fiber formation process was triggered by the applied extensional strain caused by pulling the microneedle away from the droplet. The top image shows the aligned fibrils being drawn into the fiber. The image below shows a magnification of the white box. Here, it is clearly observed that the fibrils begin in the necking region. The white arrowheads denote where the extensional strain rate likely reached the critical value required to initiate fibrillogenesis. (FI) Image sequence demonstrates the repair of a collagen fiber (full video in Movie S4). (F) End of the collagen fiber segment is identified by the white arrow. (G-I) Fiber segment end 
was placed into the droplet and drawn out, initiating a repair site (white arrow) and the continuation of new fiber formation. 

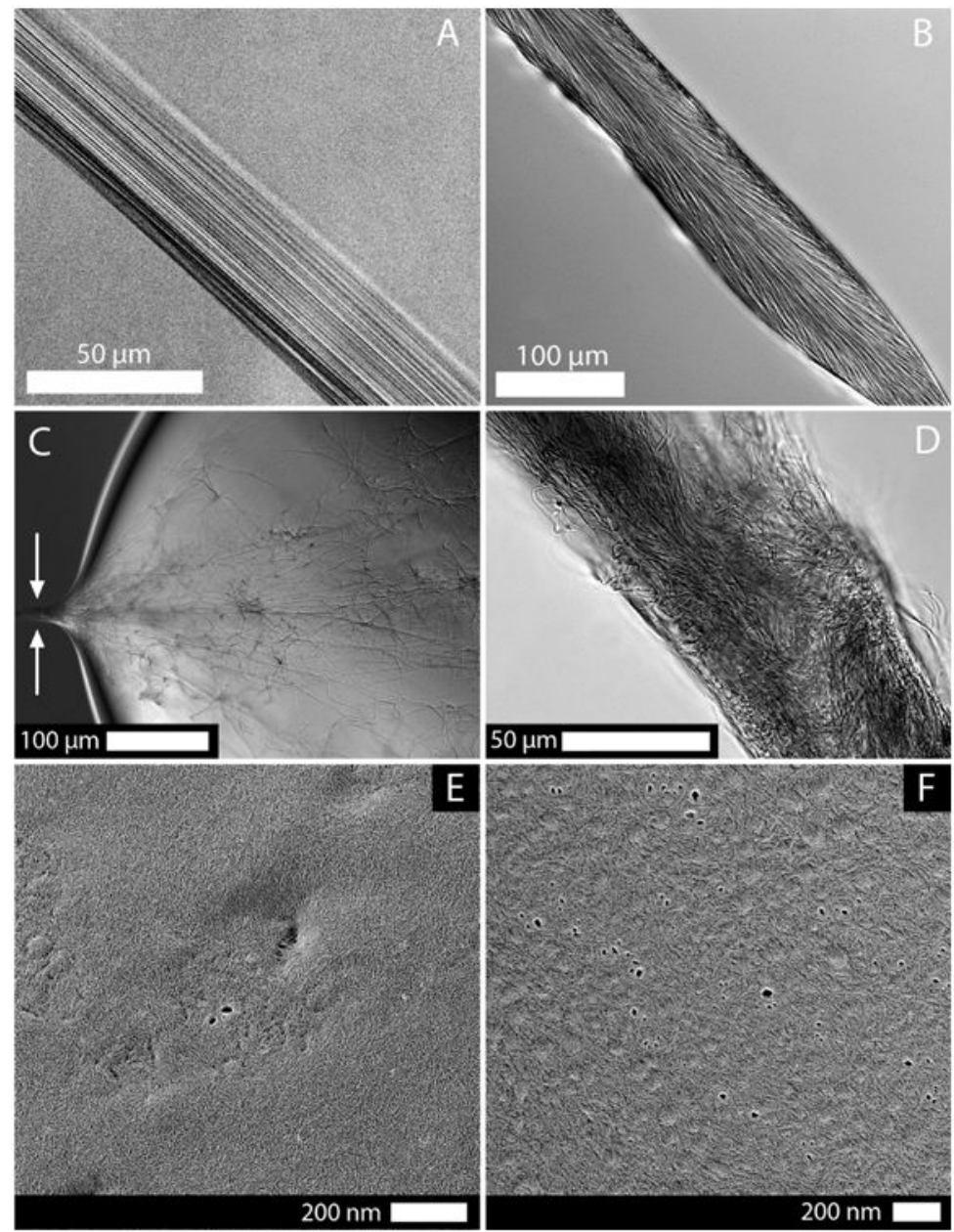

Figure 2.

Midsection of fibers pulled from solutions of (A) atelo-collagen and (B) telo-collagen observed under $1 \times$ phosphate-buffered saline (PBS). (C) Drawing a fiber from a prepolymerized droplet of telo-collagen (held under silicone oil to prevent evaporation and maintain $\mathrm{pH}$ and ionic strength). Preassembled collagen fibrils adhered to a glass microneedle and allowed a fiber to be drawn from the assembled network. The arrows identify the location from where the fiber was drawn. (D) Midsection of the fiber drawn from the preformed collagen network clearly shows the disorganization and poor spatial packing that resulted from pulling out an entangled network. The fiber morphology was far different than that observed in A or B, which indicated that triggering polymerization via extensional strain was a uniquely different process than drawing a fiber from an already formed network. This observation suggests that even cellular manipulation of an already polymerized network is unlikely to result in highly aligned fibrillar structures without further fibril modification. ${ }^{42}$ TEM images of the (E) atelo- and (F) telo-collagen droplet surface (preserved by quick freezing then replicated following deep-etching by rotary shadowing with platinum) at the time when a fiber would be drawn (150 s). Generally, the surfaces of the collagen droplet were enriched with a high concentration of monomers that have not yet assembled into larger hierarchical structures. 

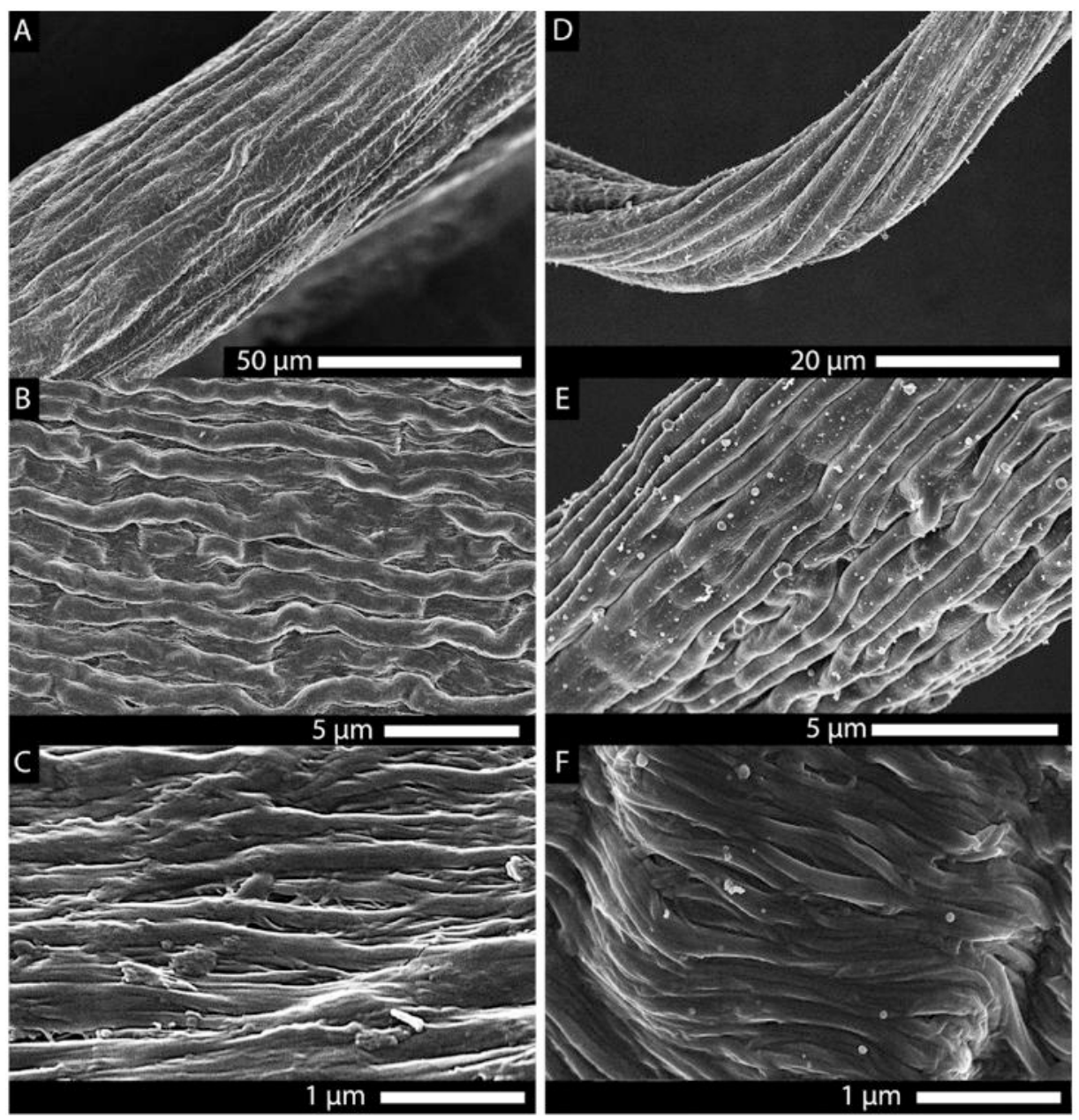

Figure 3.

Scanning electron images of the spontaneously formed hierarchical substructure of the drawn collagen fibers. (A-C) Structure of a drawn telo-collagen fiber at progressively increasing magnification. (D-F) Drawn telo-fiber from a solution that contained 2\% decorin. Both fibers displayed an impressive hierarchical morphology reminiscent of native tendon. However, the effect of the addition of decorin (a small leucine-rich proteoglycan found in the ECM, known to affect collagen fibril morphology) was apparent at the level of the individual collagen fibrils [(C) no decorin vs $(\mathrm{F})$ decorin]. As seen in vivo, decorin appears to reduce the number of lateral associations between the fibrils in vitro, as well. 

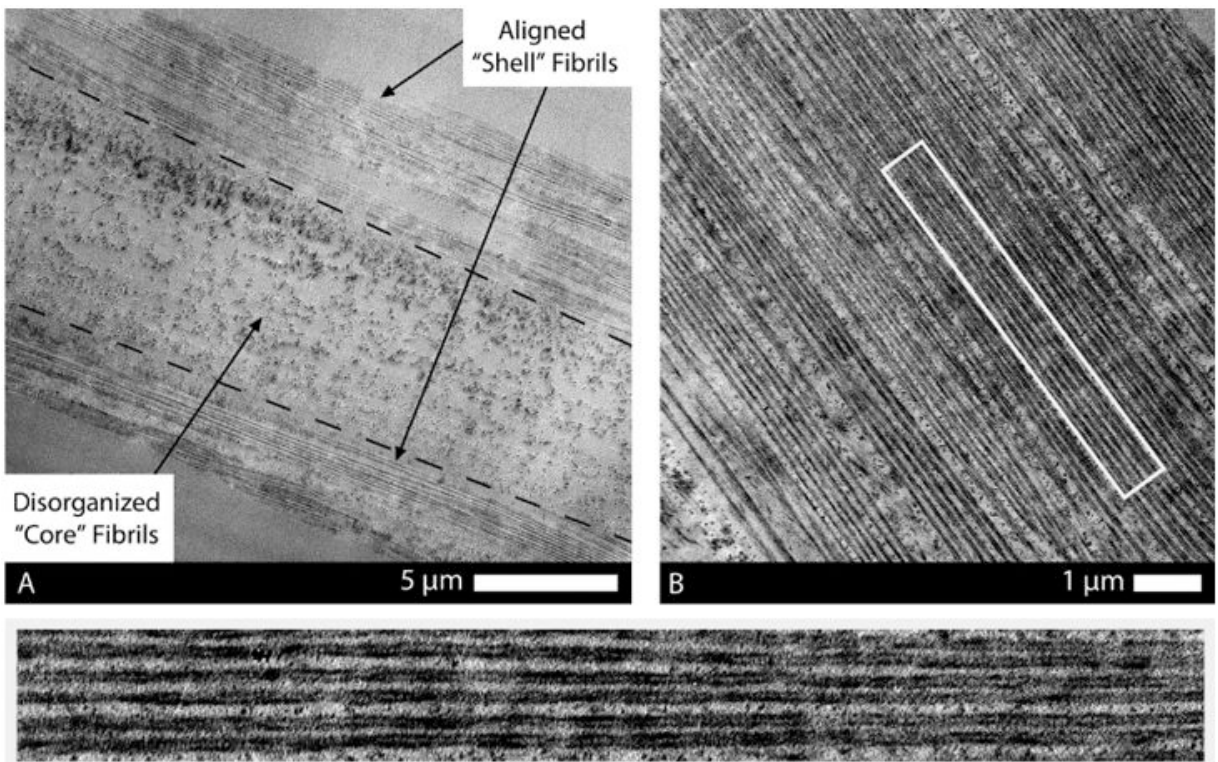

C

$500 \mathrm{~nm}$
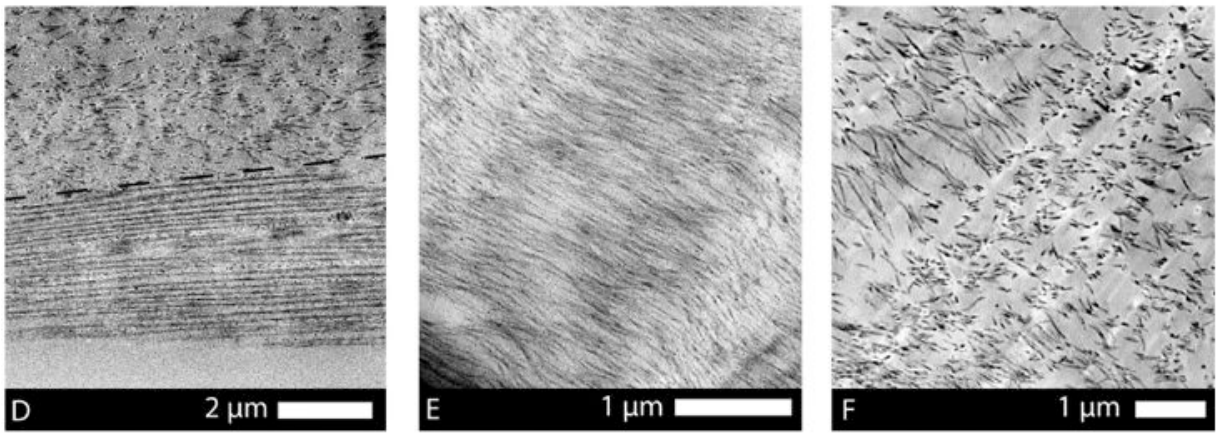

Figure 4.

TEM thin sections of drawn atelo-fibers showing their typical shell, transition, and core structure. (A) Longitudinal section of the fiber depicts the peripheral shell of highly organized fibrils and the poorly organized core of the fiber. (B) Striking alignment of fibrils in the shell, in the direction of the applied strain, was highly mimetic of native tendon morphology. (C) High magnification of the rectangular region (outlined in image B) shows the discrete, uniform diameter, consistent spacing, and continuous nature of the fibrils in the shell where the extensional strain was highest. (D) Transition from high density, continuous fibrils to randomly arranged, sparse, shorter fibrils was sometimes abrupt (typically in thinner regions of the fiber). (E) In sections of fibers with a larger diameter (>20 $\mu \mathrm{m})$, a transitional region was present, comprising short and wavy fibrils that were oriented in the general direction of the extensional strain. (F) Core of the fibril comprised discrete, isotropic fibrils with low spatial density. 

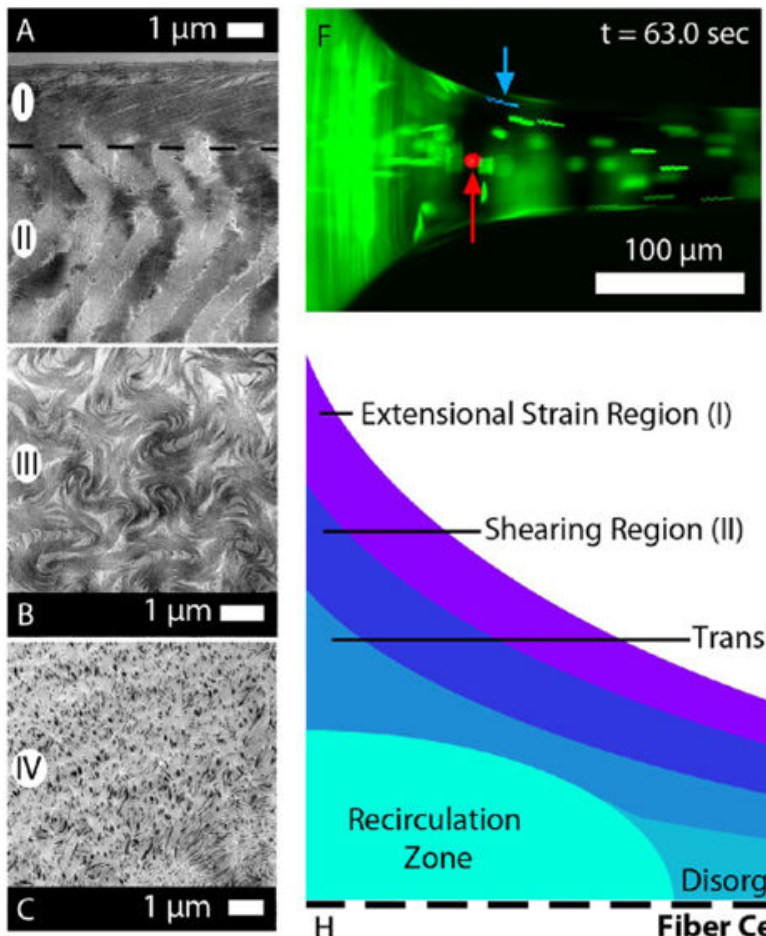

Extensional Strain Region (I)
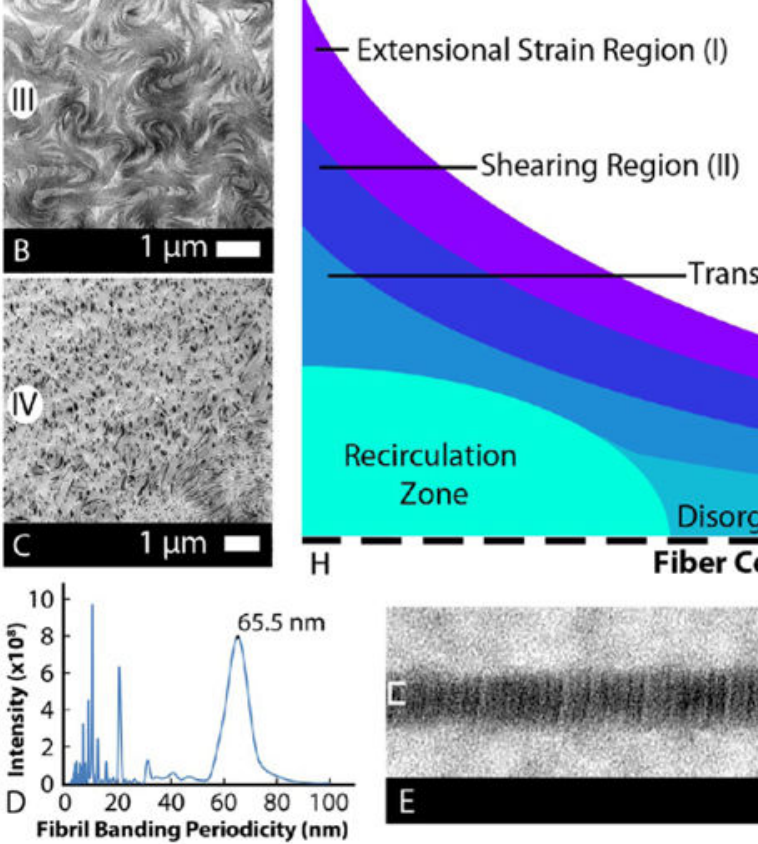

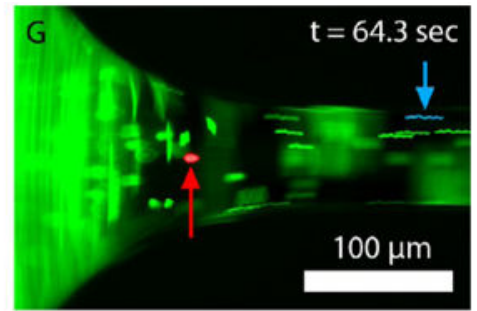

$\sim 15$ $\mathrm{mg} / \mathrm{ml}$

$\sim 4.5$

$\mathrm{mg} / \mathrm{ml}$
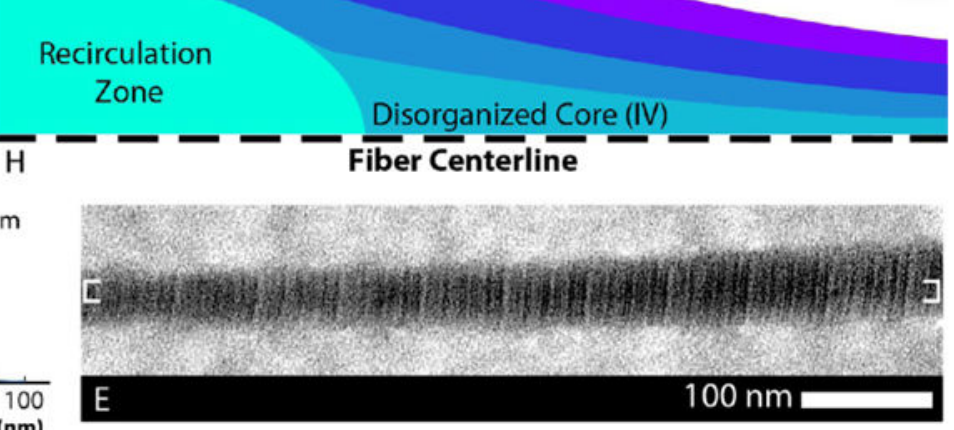

Figure 5.

Collagen concentration gradient along the depth of the droplet surface and varying extensional strain rate regimes during fiber drawing account for the multiphase fiber morphology seen in the TEM images. (A) Highest extensional strain, $0.7 \mathrm{~s}^{-1}$, was achieved on the surface of the necking region during drawing, and the corresponding structure (I) shown in A comprised highly aligned, FIC telo-collagen fibrils. Just below the surface, the collagen concentration was lower and the flow had a shear component which yielded angled, disrupted fibrillar morphology (II). (B) In the transitional region, the rotational component of the flow increased, and the further decreased collagen concentration produced a swirling morphology (III). (C) At the core of the necking region, the concentration was approximately that of the bulk concentration, $4.6 \mathrm{mg} / \mathrm{mL}$, and the resulting fiber morphology (IV) was an isotropic matrix. (D,E) Molecular positioning within the fibrils was also investigated using TEM for banding periodicity. (D) Bracketed region in E was analyzed using power spectral analysis to measure the banding periodicity, and the dominant frequency correlated to a $65.5 \mathrm{~nm}$ banding periodicity. (F,G) Images taken during the drawing of a telo-fiber with fluorescent beads in the solution (see Movie S3). The fluorescent bead highlighted in red remained stagnant, while the bead highlighted in blue accelerated along the hyperbolic geometry and entered the fiber. The full video identifies a recirculation zone at the base of the necking region, which explains the morphology 
observed in A-C. (H) Schematic characterizes proposed rheological flow regimes in the necking region, based on fluorescent particle velocity profiles and concentration measurements throughout the droplet (see Figure S7). 


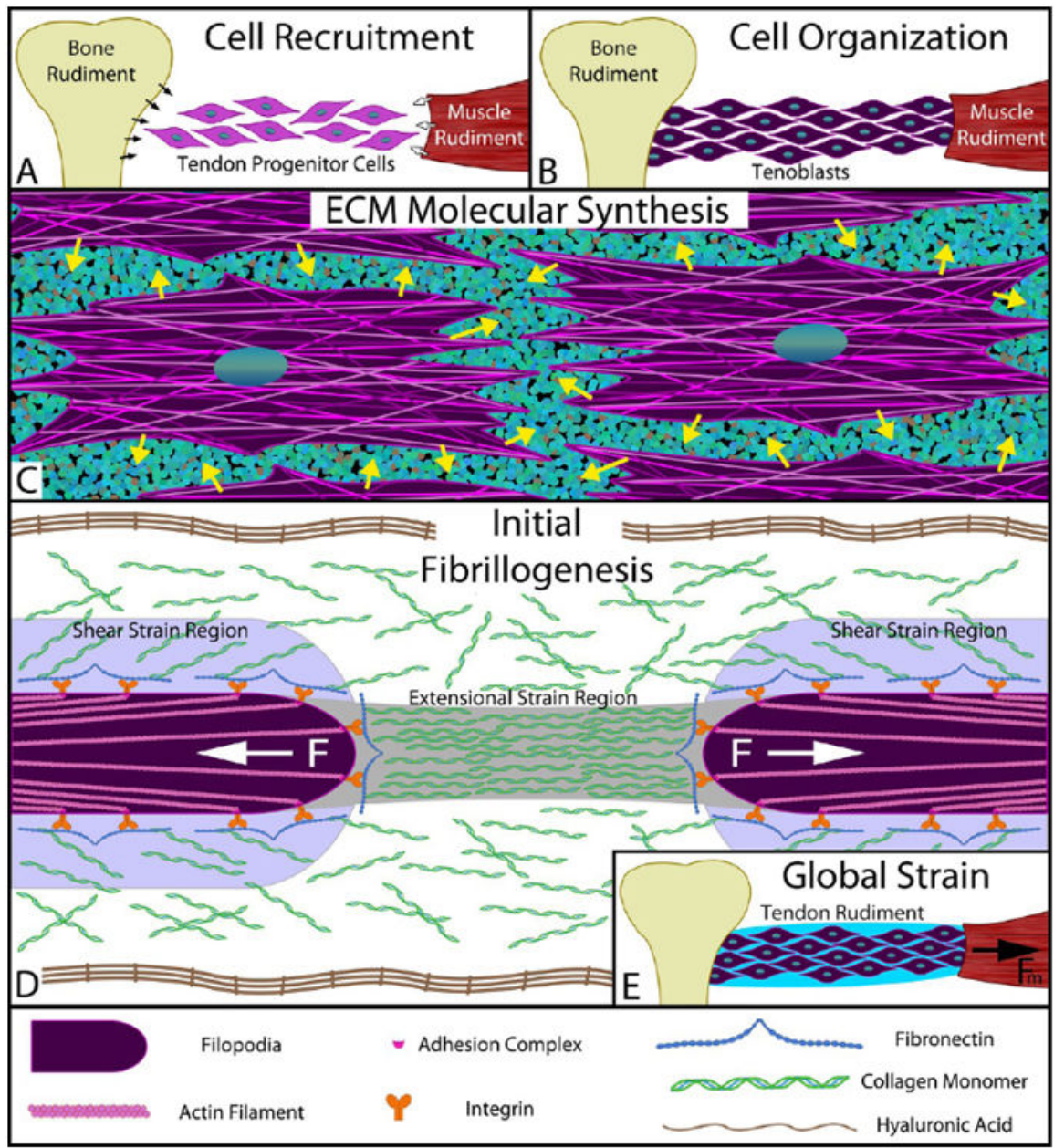

Figure 6.

Simplified model of early stage of tendon morphogenesis in vivo. (A) Recruitment: Tendon progenitor cells express signaling molecules, such as TGF $\beta,{ }^{65}$ which recruit additional cells from the cartilaginous bone rudiment (black arrows) and the muscle rudiment (white arrows). (B) Migration/organization: Recruited cells migrate into the prospective tendon space between the bone and muscle rudiments, organize to the long axis of the tendon, and take on a "tenoblastic" identity. (C) Synthesis: "Tenoblast" cells discharge structural molecules into the extracellular space (e.g., collagen monomers, fibronectin, elastin, and proteoglycans) as well as hyaluronic acid to molecularly crowd the environment surrounding the cells. (D) Discrete segment fibrillogenesis: Filopodia on the fibroblasts express collagenbinding complexes (e.g., a5 $\beta 1$-fibronectin-collagen) and exert a contractile force via nonmuscle myosin II. The flow field around the retracting filopodia creates both extensional and shear strains within the surrounding chemical milieu, which align the crowded collagen molecules and trigger FIC to produce fibril segments. Our data indicate that the retraction can produce extensional strains sufficient to cause FIC for $\sim 10 \mu \mathrm{m}$ (see Figure S8a). Each filopodia retracts until a stalling force resistance is met or until complete contraction of the actin filament occurs, at which point an invagination in the cell membrane may result. During this phase of morphogenesis, local cell contraction should manifest in the form of 
short, disconnected fibrils as seen in early tendon morphogenesis. ${ }^{76}$ (E) Drive to continuity: Tissue strains, imposed through the contraction of the muscle rudiment, preferentially induce amplified extensional strain rates (see eq 1 and Supporting Information on extensional strain rate magnification) in the gaps between cells and between fibril segments. This triggers the formation of additional collagen fibrils precisely where they are required for connectivity. As fibril segments fuse, gaps shrink and the extensional strains are further amplified. FIC continues to occur under the continually increasing extensional strain rates until continuity of load-bearing structure is converged upon. The model proposed is consistent with most observations of tendon morphogenesis, particularly with regard to fibrillogenesis. 


\section{Table 1}

\begin{tabular}{|c|c|c|}
\hline$D$ & absolute position $(\mu \mathrm{m})$ & velocity $(\mu \mathrm{m} / \mathrm{s})$ \\
\hline & 1000 & 20 \\
\hline & 2000 & 90 \\
\hline & 11000 & 240 \\
\hline & 13000 & 130 \\
\hline
\end{tabular}

